\title{
RECONSTRUCTION OF ISOTROPIC CONDUCTIVITIES FROM NON SMOOTH ELECTRIC FIELDS
}

\author{
MARC BRIANE*
}

\begin{abstract}
In this paper we study the isotropic realizability of a given non smooth gradient field $\nabla u$ defined in $\mathbb{R}^{d}$, namely when one can reconstruct an isotropic conductivity $\sigma>0$ such that $\sigma \nabla u$ is divergence free in $\mathbb{R}^{d}$. On the one hand, in the case where $\nabla u$ is non-vanishing, uniformly continuous in $\mathbb{R}^{d}$ and $\Delta u$ is a bounded function in $\mathbb{R}^{d}$, we prove the isotropic realizability of $\nabla u$ using the associated gradient flow combined with the DiPerna, Lions approach for solving ordinary differential equations in suitable Sobolev spaces. On the other hand, in the case where $\nabla u$ is piecewise regular, we prove roughly speaking that the isotropic realizability holds if and only if the normal derivatives of $u$ on each side of the gradient discontinuity interfaces have the same sign. Some examples of conductivity reconstruction are given.
\end{abstract}

Mathematics Subject Classification. 35B27, 78A30, 37C10

Received June 18, 2017. Accepted February 9, 2018.

\section{INTRODUCTION}

In Electrophysics there are some constraints implicitly satisfied by the electric field in a prescribed conductive material. For example, Alessandrini and Nesi [2] have shown that a smooth periodic electric field cannot vanish in dimension two, while it may vanish in dimension three as proved in [3, 6]. This three-dimensional specificity of the electric field allows us to derive a surprising property of the Hall effect: the sign of the Hall voltage is indeed inverted in a three-dimensional metamaterial inspired by a chain mail armor. The anomalous Hall effect has been first proved theoretically in [5], then it has been simplified and validated experimentally in [12]. Very recently it has been emphasized simultaneously in Physics Today [14] and Nature [15].

Conversely, starting from a regular gradient field $\nabla u \neq 0$ in $\mathbb{R}^{d}\left({ }^{1}\right)$ the natural inverse problem is to reconstruct from $\nabla u$ a possibly isotropic conductivity $\sigma$ which satisfies the conductivity equation

$$
\operatorname{div}(\sigma \nabla u)=0 \quad \text { in } \mathbb{R}^{d} .
$$

The gradient field $\nabla u$ is then said to be isotropically realizable. This reconstruction problem has been widely studied in the literature in terms of uniqueness, stability or instability, and algorithms of approximate solution

\footnotetext{
Keywords and phrases: Isotropic conductivity, electric field, conductivity reconstruction, gradient flow, triangulation.

University of Rennes, INSA Rennes, CNRS, IRMAR - UMR 6625, 35000 Rennes, France.

* Corresponding author: mbriane@insa-rennes.fr

${ }^{1}$ When $d=2, \nabla u \neq 0$ in the periodic case (see [2]), otherwise it is obvious that there exist solutions with $\nabla u$ vanishing somewhere. A treatment of such cases can be found in [1]. The case $d=3$ is quite different, since $\nabla u$ may vanish somewhere in the periodic case (see [6]). 
(see, e.g., $[10,13]$ and the references therein). The isotropy constraint is actually appropriate in Materials Science, since composite materials are built from isotropic phases. Moreover, the homogeneous conductivity equation (1.1) is satisfied by the local electric fields in periodic composites. We have proved in [7] that any gradient field $\nabla u$ which is non-vanishing and regular is isotropically realizable in $\mathbb{R}^{d}$. The main ingredient of this construction is the associated gradient flow

$$
\left\{\begin{aligned}
\frac{\partial X}{\partial t}(t, x) & =\nabla u(X(t, x)) \quad \text { for } t \in \mathbb{R}, x \in \mathbb{R}^{d} . \\
X(0, x) & =x
\end{aligned}\right.
$$

The dynamical approach of [7] forces the regularity $u \in C^{3}\left(\mathbb{R}^{d}\right)$. However, this smoothness is not compatible with most of composite materials where the gradient is only piecewise regular (for instance regular in each phase of the material). The purpose of the present work is to extend the results of [7] to less regular gradient fields. To this end, we study two independent cases which are respectively developed in Sections 2 and 3.

In Section 2 we assume that the gradient field $\nabla u$ is continuous in $\mathbb{R}^{d}$. The idea is to modify the strategy of [7] applying the celebrated approach of DiPerna and Lions [9] for solving ordinary differential equations in suitable Sobolev spaces. More precisely, we prove (see Thm. 2.1) that any gradient field $\nabla u$ in $W_{\text {loc }}^{1,1}\left(\mathbb{R}^{d}\right)^{d}$ is isotropically realizable in $\mathbb{R}^{d}$ if

$$
\nabla u \text { is uniformly continuous in } \mathbb{R}^{d}, \quad \Delta u \in L^{\infty}\left(\mathbb{R}^{d}\right) \quad \text { and } \quad \inf _{\mathbb{R}^{d}}|\nabla u|>0 .
$$

Moreover, any positive function $\sigma \in L_{\mathrm{loc}}^{\infty}\left(\mathbb{R}^{d}\right)$ with $\sigma^{-1} \in L_{\mathrm{loc}}^{\infty}\left(\mathbb{R}^{d}\right)$ is shown to be a suitable conductivity if and only if roughly speaking (see Rem. 2.3) there exists $E$, a set of Lebesgue measure zero, such that

$$
\frac{\sigma(x)}{\sigma(X(t, x))}=\exp \left(\int_{0}^{t} \Delta u(X(s, x)) \mathrm{d} s\right), \quad \forall t \in \mathbb{R}, \forall x \in \mathbb{R}^{d} \backslash E,
$$

where $X(\cdot, x)$ is the gradient flow (1.2). Assumption (1.3) improves significantly the regularity $u \in C^{3}\left(\mathbb{R}^{d}\right)$ which is needed in [7]. But the price to pay is that the reconstruction of an appropriate conductivity is much more delicate. In particular, by [9] the flow $X(\cdot, x)$ of $(1.2)$ is only continuous for almost everywhere $x \in \mathbb{R}^{d}$. However, condition (1.3) is not still satisfactory since it excludes most of the Lipschitz continuous potentials $u$ which naturally arise in composite materials.

In Section 3 we study the case of a piecewise regular gradient $\nabla u$ in a domain $\Omega$ of $\mathbb{R}^{d}$ composed by $n$ "generalized" polyhedra $\Omega_{k}$ (i.e. obtained from polyhedra through a smooth diffeomorphism). The continuous potential $u$ agrees in each set $\Omega_{k}$ to a function $u_{k} \in C^{2}\left(\overline{\Omega_{k}}\right)$ such that the trajectories of (1.2) flow from an inflow boundary face (on which the outer normal derivative of $u_{k}$ is negative) to an outflow boundary face (on which the outer normal derivative of $u_{k}$ is positive), while the other boundary faces are tangential to $\nabla u_{k}$ (see Fig. 1). We prove (see Thm. 3.7) that there exists a piecewise continuous conductivity $\sigma$ solution to equation (1.1) if and only if for any contiguous polyhedra $\Omega_{j}$ and $\Omega_{k}$ of $\Omega$, the normal derivatives satisfy the condition

$$
\frac{\partial u_{j}}{\partial \nu}=\frac{\partial u_{k}}{\partial \nu}=0 \text { on } \partial \Omega_{j} \cap \partial \Omega_{k} \quad \text { or } \quad \frac{\partial u_{j}}{\partial \nu} \frac{\partial u_{k}}{\partial \nu}>0 \text { on } \partial \Omega_{j} \cap \partial \Omega_{k} .
$$

In the first case the common boundary face $\partial \Omega_{j} \cap \partial \Omega_{k}$ is tangential to the gradient, while in the second case $\partial \Omega_{j} \cap \partial \Omega_{k}$ is an inflow (resp. outflow) face of $\Omega_{j}$ and an outflow (resp. inflow) face of $\Omega_{k}$. Actually, the picture is a little more constrained: We need to consider a so-called $\nabla u$-admissible domain $\Omega$ (see Def. 3.5). Figure 2 represents a $\nabla u$-admissible set, and Figure 3 represents a non-admissible one.

We construct step by step a suitable piecewise conductivity $\sigma$ such that $\sigma=\sigma_{k}$ in $\Omega_{k}$ as follows. If $\sigma_{j}$ is already constructed in $\Omega_{j}$, by $[4,16]$ (see Prop. 3.1 for details) there exists a unique positive function 
$\sigma_{k} \in C^{1}\left(\overline{\Omega_{k}}\right)$ solution to the equation $\operatorname{div}\left(\sigma_{k} \nabla u_{k}\right)=0$ in $\Omega_{k}$, and equal on the inflow or outflow face $\partial \Omega_{j} \cap \partial \Omega_{k}$ to the boundary value $\gamma_{k} \in C\left(\partial \Omega_{j} \cap \partial \Omega_{k}\right)$ which ensures by virtue of (1.5) the flux continuity condition

$$
\sigma_{j} \frac{\partial u_{j}}{\partial \nu}=\gamma_{k} \frac{\partial u_{k}}{\partial \nu} \quad \text { on } \partial \Omega_{j} \cap \partial \Omega_{k}
$$

So, the piecewise continuous function $\sigma=\sigma_{k}$ in $\Omega_{k}$ is a solution to the equation $\operatorname{div}(\sigma \nabla u)=0$ in the distributional sense of $\Omega$.

In Section 4 the results of Section 3 are illustrated by the case of piecewise constant gradients in some triangulation (see Fig. 4), and the case of the gradient of a function $u \in C\left(\mathbb{R}^{d}\right)$ defined by $u(x):=g_{ \pm}\left(x_{1}\right)+$ $f\left(x_{2}, \ldots, x_{d}\right)$ in each half-space $\left\{ \pm x_{1}>0\right\}$.

\section{Notation}

- $\operatorname{int}(A)$ denotes the interior of a subset $A$ of $\mathbb{R}^{d}$.

- $C(A)$ denotes the set of continuous functions in a topological space $A$.

- $C^{k}(A)$ denotes the space of $k$-differentiable functions in a subset $A$ of $\mathbb{R}^{d}$, and $C_{c}^{k}(A)$ denotes the subspace of $C^{k}(A)$ composed of functions with compact support in $A$.

- $\mathscr{D}^{\prime}(\Omega)$ denotes the distributions space in an open set $\Omega$ of $\mathbb{R}^{d}$.

- $c$ denotes a positive constant which may vary from line to line.

\section{CASE Where the GRAdient Field is CONTINUOUS}

For $u \in W_{\text {loc }}^{2,1}\left(\mathbb{R}^{d}\right)$, the gradient flow $X=X(t, x)$ associated with $\nabla u$ is defined (if possible) by

$$
\left\{\begin{aligned}
\frac{\partial X}{\partial t}(t, x) & =\nabla u(X(t, x)) \\
X(0, x) & =x
\end{aligned} \quad \text { for } t \in \mathbb{R}, x \in \mathbb{R}^{d} .\right.
$$

Theorem 2.1. Let $u: \mathbb{R}^{d} \rightarrow \mathbb{R}$ be a function satisfying

$$
u \in W_{\text {loc }}^{2,1}\left(\mathbb{R}^{d}\right), \quad \nabla u \text { is uniformly continuous in } \mathbb{R}^{d}, \quad \Delta u \in L^{\infty}\left(\mathbb{R}^{d}\right), \quad \inf _{\mathbb{R}^{d}}|\nabla u|>0 .
$$

Then, there exists a positive function $\sigma \in L_{\mathrm{loc}}^{\infty}\left(\mathbb{R}^{d}\right)$ with $\sigma^{-1} \in L_{\mathrm{loc}}^{\infty}\left(\mathbb{R}^{d}\right)$, solution to the conductivity equation

$$
\operatorname{div}(\sigma \nabla u)=0 \quad \text { in } \mathscr{D}^{\prime}\left(\mathbb{R}^{d}\right),
$$

the flow $X(\cdot, x)$ is well defined by (2.1) for a.e. $x \in \mathbb{R}^{d}$, and $\sigma$ satisfies the following: for any $t \in \mathbb{R}$, there exists a set $E_{t}$, of Lebesgue measure zero depending on $t$, such that

$$
\frac{\sigma(x)}{\sigma(X(t, x))}=\exp \left(\int_{0}^{t} \Delta u(X(s, x)) \mathrm{d} s\right), \quad \forall x \in \mathbb{R}^{d} \backslash E_{t} .
$$

Conversely, if there exists $E$, a set of Lebesgue measure zero, and a positive function $\sigma$ in $L_{\mathrm{loc}}^{\infty}\left(\mathbb{R}^{d}\right)$ such that

$$
\frac{\sigma(x)}{\sigma(X(t, x))}=\exp \left(\int_{0}^{t} \Delta u(X(s, x)) \mathrm{d} s\right)
$$

holds for any $t \in \mathbb{R}$ and any $x \in \mathbb{R}^{d} \backslash E$, then $\sigma$ is solution to equation (2.3). 
Remark 2.2. Assumptions (2.2) replace the smoothness $u \in C^{3}\left(\mathbb{R}^{d}\right)$ which is needed in [7].

Remark 2.3. The set $E$ of Lebesgue measure zero where formula (2.5) is not satisfied by $x$ does not depend on $t$, while the set $E_{t}$ does depend on $t$ in formula (2.4). Hence, formula (2.5) is stronger than (2.4). Both formulas are equivalent if for instance $X, \Delta u$ and $\sigma$ are continuous.

Proof of Theorem 2.1. Let $\left(\rho_{n}\right)_{n \geq 1}$ be a sequence of mollifiers satisfying

$$
\rho_{n} \in C^{\infty}\left(\mathbb{R}^{d}\right), \quad \operatorname{supp}\left(\rho_{n}\right) \subset B(0,1 / n), \quad \rho_{n} \geq 0, \quad \int_{\mathbb{R}^{d}} \rho_{n}(x) \mathrm{d} x=1 .
$$

Denote $u_{n}:=\rho_{n} * u \in C^{\infty}\left(\mathbb{R}^{d}\right)$. Since by (2.2) $\nabla u$ is uniformly continuous in $\mathbb{R}^{d}$, the sequence $\nabla u_{n}=\rho_{n} * \nabla u$ converges uniformly to $\nabla u$ in $\mathbb{R}^{d}$. Hence, by the last inequality of (2.2) there exists a constant $m>0$ such that

$$
\inf _{\mathbb{R}^{d}}\left|\nabla u_{n}\right| \geq m>0 \quad \text { for } n \text { large enough. }
$$

Let $X_{n}(t, x)$ be the flow associated with $\nabla u_{n}$ defined by

$$
\left\{\begin{aligned}
\frac{\partial X_{n}}{\partial t}(t, x) & =\nabla u_{n}(X(t, x)) \\
X_{n}(0, x) & =x
\end{aligned} \quad \text { for } t \in \mathbb{R}, x \in \mathbb{R}^{d} .\right.
$$

By (2.7) the regular case of Theorem 2.15 in [7] shows that there exists a unique function $\tau_{n}$ in $C^{\infty}\left(\mathbb{R}^{d}\right)$ satisfying

$$
u_{n}\left(X_{n}\left(\tau_{n}(x), x\right)\right)=0, \quad \forall x \in \mathbb{R}^{d},
$$

and that, denoting

$$
\sigma_{n}(x):=\exp \left(\int_{0}^{\tau_{n}(x)} \Delta u_{n}\left(X_{n}(s, x)\right) \mathrm{d} s\right) \quad \text { for } x \in \mathbb{R}^{d}
$$

we have

$$
\operatorname{div}\left(\sigma_{n} \nabla u_{n}\right)=0 \quad \text { in } \mathbb{R}^{d},
$$

and

$$
\frac{\sigma_{n}(x)}{\sigma_{n}\left(X_{n}(t, x)\right)}=\exp \left(\int_{0}^{t} \Delta u_{n}\left(X_{n}(s, x)\right) \mathrm{d} s\right), \quad \forall x \in \mathbb{R}^{d}, \forall t \in \mathbb{R}
$$

The main difficulty is now to pass to the limit $n \rightarrow \infty$ in equations (2.10), (2.11), (2.12). To this end, we will use the approach of DiPerna and Lions [9] for solving ordinary differential equations in Sobolev spaces. First of all, note that by condition (2.2) the field $b:=\nabla u$ satisfies the condition (49) and (70) of [9], i.e.

$$
\frac{b}{1+|x|} \in L^{\infty}\left(\mathbb{R}^{d}\right), \quad b \in W_{\mathrm{loc}}^{1,1}\left(\mathbb{R}^{d}\right)^{d} \quad \text { and } \quad \operatorname{div} b \in L^{\infty}\left(\mathbb{R}^{d}\right)
$$

since any uniformly continuous function $f(x)$ in $\mathbb{R}^{d}$ is bounded by an affine function of $|x|$. Hence, by virtue of Theorem III.2 in [9], the flow $X_{n}(\cdot, x)$ converges in $C_{\text {loc }}(\mathbb{R})$ to the unique flow $X(\cdot, x) \in C^{1}\left(\mathbb{R}^{d}\right)^{d}$ defined by 
(2.1) for a.e. $x \in \mathbb{R}^{d}$. Moreover, $X$ satisfies the semi-group property: for any $t \in \mathbb{R}$, there exists a set $E_{t}$, of Lebesgue measure zero depending on $t$, such that

$$
X(s+t, x)=X(s, X(t, x)), \quad \forall s \in \mathbb{R}, \forall x \in \mathbb{R}^{d} \backslash E_{t} .
$$

The image measure $\lambda_{X}(t)$, for $t \in \mathbb{R}$, of the Lebesgue measure $\lambda$ by $X(t, \cdot)$, i.e. defined by

$$
\int_{\mathbb{R}^{d}} \varphi \mathrm{d} \lambda_{X}(t)=\int_{\mathbb{R}^{d}} \varphi(X(t, x)) \mathrm{d} x, \quad \forall \varphi \in C_{c}\left(\mathbb{R}^{d}\right),
$$

has a density in $r(t, \cdot) \in L^{\infty}\left(\mathbb{R}^{d}\right)$ with respect to the Lebesgue measure, which satisfies for any $t \in \mathbb{R}$,

$$
e^{-|t|\|\Delta u\|_{L^{\infty}\left(\mathbb{R}^{d}\right)}} \leq r(t, \cdot) \leq e^{|t|\|\Delta u\|_{L^{\infty}\left(\mathbb{R}^{d}\right)}} \text { a.e. in } \mathbb{R}^{d},
$$

or equivalently, for any $t \in \mathbb{R}$ and for any $\varphi \in C_{c}\left(\mathbb{R}^{d}\right), \varphi \geq 0$,

$$
e^{-|t|\|\Delta u\|_{L^{\infty}\left(\mathbb{R}^{d}\right)}} \int_{\mathbb{R}^{d}} \varphi(x) \mathrm{d} x \leq \int_{\mathbb{R}^{d}} \varphi \mathrm{d} \lambda_{X}(t) \leq e^{|t|\|\Delta u\|_{L^{\infty}\left(\mathbb{R}^{d}\right)}} \int_{\mathbb{R}^{d}} \varphi(x) \mathrm{d} x .
$$

We will need the following result satisfied by the flows $X_{n}$ and $X$.

\section{Lemma 2.4.}

(i) If $f \in L_{\mathrm{loc}}^{1}\left(\mathbb{R}^{d}\right)$ then $f \circ X \in L_{\mathrm{loc}}^{1}\left(\mathbb{R} \times \mathbb{R}^{d}\right)$.

(ii) Let $f \in L_{\text {loc }}^{1}\left(\mathbb{R}^{d}\right)$, let $K$ be a compact of $\mathbb{R}^{d}$, and let $I$ be a bounded interval of $\mathbb{R}$. Then, we have

$$
\lim _{n \rightarrow \infty} \int_{K} \int_{I}\left|f\left(X_{n}(s, x)\right)-f(X(s, x))\right| \mathrm{d} s \mathrm{~d} x=0 .
$$

(iii) Let $f_{n}$ be a non-negative sequence of $L_{\text {loc }}^{1}\left(\mathbb{R}^{d}\right)$ which converges strongly to 0 in $L_{\mathrm{loc}}^{1}\left(\mathbb{R}^{d}\right)$, let $K$ be a compact of $\mathbb{R}^{d}$, and let $I$ be a bounded interval of $\mathbb{R}$. Then, we have

$$
\lim _{n \rightarrow \infty} \int_{K} \int_{I} f_{n}\left(X_{n}(s, x)\right) \mathrm{d} s \mathrm{~d} x=0 .
$$

(iv) Let $F \in L^{p}\left(\mathbb{R}^{d}\right)^{N}$ for $N \in \mathbb{N}, p \in[1, \infty)$, let $G \in L^{p^{\prime}}\left(\mathbb{R}^{d}\right)^{N}$ with compact support, where $p^{\prime}$ is the conjugate exponent of $p$, and let $\rho_{n}$ be a sequence in $C_{c}^{\infty}(\mathbb{R})$ satisfying $(2.6)$ with $d=1$. Then, we have

$$
\lim _{n \rightarrow \infty} \int_{\mathbb{R}^{d}} \int_{\mathbb{R}} \rho_{n}(s) F(X(s, x)) \cdot G(x) \mathrm{d} s \mathrm{~d} x=\int_{\mathbb{R}^{d}} F(x) \cdot G(x) \mathrm{d} x .
$$

The proof is divided in five steps.

First step: Convergence of the sequence $\tau_{n}$ defined by (2.9).

On the one hand, since by (2.2) there exists $E$, a set of Lebesgue measure zero, such that for any $x \in \mathbb{R} \backslash E$,

$$
\frac{\mathrm{d}}{\mathrm{d} t}\left(u(X(t, x))=|\nabla u|^{2}(X(t, x)) \geq \inf _{\mathbb{R}^{d}}|\nabla u|^{2}>0, \quad \forall t \in \mathbb{R},\right.
$$

there exists a unique $\tau(x) \in \mathbb{R}$ such that

$$
u(X(\tau(x), x))=0 \quad \text { for a.e. } x \in \mathbb{R}^{d} .
$$


On the other hand, by (2.9) we have

$$
\left|u_{n}(x)\right|=\left|u_{n}(x)-u_{n}\left(X_{n}\left(\tau_{n}(x), x\right)\right)\right|=\left.\left|\int_{0}^{\tau_{n}(x)}\right| \nabla u_{n}\right|^{2}\left(X_{n}(t, x)\right) \mathrm{d} s\left|\geq m^{2}\right| \tau_{n}(x) \mid \quad \text { a.e. } x \in \mathbb{R}^{d} .
$$

Hence, since $u_{n}$ converges uniformly to $u$ in any compact set $K$ of $\mathbb{R}^{d}$, the sequence $\tau_{n}$ is bounded in $L^{\infty}(K)$. Let $x \in \mathbb{R}^{d}$ be satisfying (2.22). Up to a subsequence still denoted by $n, \tau_{n}(x)$ converges to some $\tau_{x}$ in $\mathbb{R}$. Using the uniform convergence of $X_{n}(\cdot, x)$ to $X(\cdot, x)$ and passing to the limit in equality $(2.9)$ we get that $u\left(X\left(\tau_{x}, x\right)\right)=0$, which by uniqueness of $\tau(x)$ implies that $\tau_{x}=\tau(x)$. Therefore, we obtain for the whole sequence

$$
\lim _{n \rightarrow \infty} \tau_{n}(x)=\tau(x) \text { for a.e. } x \in \mathbb{R}^{d} .
$$

Since $\tau$ is measurable and $\Delta u \circ X \in L_{\text {loc }}^{1}\left(\mathbb{R} \times \mathbb{R}^{d}\right)$ by Lemma 2.4, applying Fubini's theorem to the function $(t, x) \mapsto 1_{[0, \tau(x)]}(t) \Delta u(X(t, x))$ in $L_{\mathrm{loc}}^{1}\left(\mathbb{R} \times \mathbb{R}^{d}\right)$, we can define the measurable function $\sigma$ by

$$
\sigma(x):=\exp \left(\int_{0}^{\tau(x)} \Delta u(X(s, x)) \mathrm{d} s\right) \text { for a.e. } x \in \mathbb{R}^{d} .
$$

Second step: Strong convergence of the sequence $w_{n}:=\ln \sigma_{n}$ to $w:=\ln \sigma$ in $L_{\text {loc }}^{1}\left(\mathbb{R}^{d}\right)$.

Let $K$ be a compact set of $\mathbb{R}^{d}$. We have

$$
\begin{aligned}
\int_{K}\left|w_{n}(x)-w(x)\right| \mathrm{d} x & \leq \int_{K}\left|\int_{0}^{\tau_{n}(x)}\right| \Delta u\left(X_{n}(s, x)\right)-\Delta u(X(s, x))|\mathrm{d} s| \mathrm{d} x & =: E_{n}^{1} \\
& +\int_{K}\left|\int_{0}^{\tau_{n}(x)}\right| \Delta u_{n}-\Delta u\left|\left(X_{n}(s, x)\right) \mathrm{d} s\right| \mathrm{d} x & =: E_{n}^{2} \\
& +\int_{K}\left|\int_{\tau(x)}^{\tau_{n}(x)}\right| \Delta u(X(s, x))|\mathrm{d} s| \mathrm{d} x & =: E_{n}^{3} .
\end{aligned}
$$

Since by the first step the sequence $\tau_{n}$ is uniformly bounded in any compact set of $\mathbb{R}^{d}$, there exist a bounded interval $I$ of $\mathbb{R}$ such that

$$
E_{n}^{1} \leq \int_{K} \int_{I}\left|\Delta u\left(X_{n}(s, x)\right)-\Delta u(X(s, x))\right| \mathrm{d} s \mathrm{~d} x
$$

Hence, applying the limit (2.18) of Lemma 2.4 with $f:=\Delta u$, we get that $E_{n}^{1}$ tends to 0 . Similarly, applying (2.19) with the sequence $f_{n}:=\Delta u_{n}-\Delta u=\rho_{n} * \Delta u-\Delta u$ which converges strongly to 0 in $L_{\text {loc }}^{1}\left(\mathbb{R}^{d}\right)$, we get that $E_{n}^{2}$ tends to 0 . Finally, since $\tau_{n}$ is uniformly bounded in the compact $K$ and $\Delta u \in L^{\infty}\left(\mathbb{R}^{d}\right)$, by convergence (2.23) and the Lebesgue dominated convergence theorem we get that

$$
0 \leq E_{n}^{3} \leq c \int_{K}\left|\tau_{n}-\tau\right| \mathrm{d} x \underset{n \rightarrow \infty}{\longrightarrow} 0 .
$$

Therefore, passing to the limit $n \rightarrow \infty$ in $(2.25)$ we obtain that the sequence $w_{n}$ converges strongly to $w$ in $L_{\mathrm{loc}}^{1}\left(\mathbb{R}^{d}\right)$.

Third step: Derivation of the conductivity equation (2.3). 
By (2.10) the function $w_{n}$ is defined by

$$
w_{n}(x)=\int_{0}^{\tau_{n}(x)} \Delta u_{n}\left(X_{n}(s, x)\right) \mathrm{d} s \quad \text { for } x \in \mathbb{R}^{d} .
$$

Since by the first step $\tau_{n}$ is bounded in any compact of $\mathbb{R}^{d}$ and $\Delta u_{n}=\rho_{n} * \Delta u$ is bounded in $L^{\infty}\left(\mathbb{R}^{d}\right)$, the sequence $w_{n}$ is bounded in $L_{\mathrm{loc}}^{\infty}\left(\mathbb{R}^{d}\right)$. Hence, by the second step the sequence $\sigma_{n}=e^{w_{n}}$ converge strongly to $\sigma=e^{w}$ in $L_{\text {loc }}^{1}\left(\mathbb{R}^{d}\right)$. Moreover, the sequence $\nabla u_{n}$ converges to $\nabla u$ in $C_{\text {loc }}\left(\mathbb{R}^{d}\right)$. Therefore, passing to the limit in equation (2.11) we get that $\sigma$ is solution to the conductivity equation (2.3) in the distributions sense. Finally, both $\sigma$ and $\sigma^{-1}$ belong to $L_{\mathrm{loc}}^{\infty}\left(\mathbb{R}^{d}\right)$, since $\sigma$ is the limit in $L_{\mathrm{loc}}^{1}\left(\mathbb{R}^{d}\right)$ of the sequence $\sigma_{n}=e^{w_{n}}$ which is bounded in $L_{\mathrm{loc}}^{\infty}\left(\mathbb{R}^{d}\right)$.

Fourth step: Proof of formula (2.4).

Formula (2.12) reads as

$$
w_{n}(x)-w_{n}\left(X_{n}(t, x)\right)=\int_{0}^{t} \Delta u_{n}\left(X_{n}(s, x)\right) \mathrm{d} s, \quad \forall t \in \mathbb{R}, \forall x \in \mathbb{R}^{d} .
$$

On the one hand, writing

$$
\left|w_{n}\left(X_{n}(t, x)\right)-w(X(t, x))\right| \leq\left|w\left(X_{n}(t, x)\right)-w(X(t, x))\right|+\left|w_{n}-w\right|\left(X_{n}(t, x)\right),
$$

applying limit (2.18) with $f:=w$, and applying limit (2.19) with $f_{n}:=\left|w_{n}-w\right|$ which converges strongly to 0 in $L_{\text {loc }}^{1}\left(\mathbb{R}^{d}\right)$ by the second step, we get that

$$
w_{n}\left(X_{n}(t, \cdot)\right) \underset{n \rightarrow \infty}{\longrightarrow} w(X(t, \cdot)) \quad \text { strongly in } L_{\text {loc }}^{1}\left(\mathbb{R}^{d}\right) \text {, for any } t \in \mathbb{R} \text {. }
$$

On the other hand, let $K$ be a compact set of $\mathbb{R}^{d}$ and $t \in \mathbb{R}$. We have

$$
\begin{aligned}
& \int_{K}\left|\int_{0}^{t} \Delta u_{n}\left(X_{n}(s, x)\right) \mathrm{d} s-\int_{0}^{t} \Delta u(X(s, x)) \mathrm{d} s\right| \mathrm{d} x \\
& \leq\left|\int_{0}^{t} \int_{K}\left[\left|\Delta u\left(X_{n}(s, x)\right)-\Delta u(X(s, x))\right|+\left|\Delta u_{n}-\Delta u\right|\left(X_{n}(s, x)\right)\right] \mathrm{d} x \mathrm{~d} s\right| .
\end{aligned}
$$

Then, applying successively limit (2.18) with $f:=\Delta u$ and limit (2.19) with $f_{n}:=\left|\Delta u_{n}-\Delta u\right|$ in $[0, t] \times K$, we get that

$$
\int_{0}^{t} \Delta u_{n}\left(X_{n}(s, x)\right) \mathrm{d} s \underset{n \rightarrow \infty}{\longrightarrow} \int_{0}^{t} \Delta u(X(s, x)) \mathrm{d} s \quad \text { strongly in } L_{\mathrm{loc}}^{1}\left(\mathbb{R}^{d}\right), \text { for any } t \in \mathbb{R}
$$

Therefore, using the limits (2.28) and (2.29) in (2.27), there exists $E_{t}$, a set of Lebesgue measure zero depending on $t$, such that for any $t \in \mathbb{R}$,

$$
w(x)-w(X(t, x))=\int_{0}^{t} \Delta u(X(s, x)) \mathrm{d} s, \quad \forall x \in \mathbb{R}^{d} \backslash E_{t} .
$$

or equivalently formula (2.4).

Remark 2.5. A direct proof of (2.4) would consist in replacing $x$ by $X(t, x)$ in the definition (2.24) of $\sigma(x)$ and to use the semi-group property (2.14), to obtain the desired formula (2.4). However, since the function $\tau$ 
involving in (2.24) is only defined a.e. in $\mathbb{R}^{d}$ by (2.21), it is not clear that for an admissible point $x$ of $\tau, X(t, x)$ for $t \in \mathbb{R}$, is also an admissible point of $\tau$.

Fifth step: Formula (2.5) implies the conductivity equation (2.3).

Let $\sigma$ be a positive function in $L_{\text {loc }}^{\infty}\left(\mathbb{R}^{d}\right)$ satisfying formula (2.4). First of all by $(2.2)$ the function $b(t, x):=\nabla u(x)$ satisfies the assumptions $(*),(* *)$ of Theorem II.3.1 in [9] and assumptions (49), (70) of Theorem III.2 in [9]. Then, by virtue of Theorem II.3.1 in [9] and Theorem III.2 in [9] the function $\sigma(X(t, x))$ is solution to the transport equation

$$
\frac{\partial}{\partial t}[\sigma(X(t, x))]=\nabla u(x) \cdot \nabla_{x}[\sigma(X(t, x))] \quad \text { in } \mathscr{D}^{\prime}\left(\mathbb{R} \times \mathbb{R}^{d}\right)
$$

Moreover, taking the derivative with respect to $t$ in (2.5) (at this point (2.4) seems to be not sufficient) we have

$$
\frac{\partial}{\partial t}[\sigma(X(t, x))]=-\sigma(X(t, x)) \Delta u(X(t, x)) \quad \text { in } \mathscr{D}^{\prime}\left(\mathbb{R} \times \mathbb{R}^{d}\right) .
$$

Equating the two previous equations we get that

$$
\nabla_{x}[\sigma(X(t, x))] \cdot \nabla u(x)+\sigma(X(t, x)) \Delta u(X(t, x))=0 \quad \text { in } \mathscr{D}^{\prime}\left(\mathbb{R} \times \mathbb{R}^{d}\right) .
$$

Since $\nabla u \in W_{\text {loc }}^{1,1}\left(\mathbb{R}^{d}\right)$, the previous equation can be read as

$$
\operatorname{div}_{x}[\sigma(X(t, x)) \nabla u(x)]=\sigma(X(t, x))[\Delta u(x)-\Delta u(X(t, x))] \quad \text { in } \mathscr{D}^{\prime}\left(\mathbb{R} \times \mathbb{R}^{d}\right),
$$

which implies that for any $\varphi \in C_{c}^{\infty}(\mathbb{R})$ and $\psi \in C_{c}^{\infty}\left(\mathbb{R}^{d}\right)$,

$$
\begin{aligned}
& \int_{\mathbb{R}^{d}} \int_{\mathbb{R}} \varphi(t) \sigma(X(t, x)) \nabla u(x) \cdot \nabla \psi(x) \mathrm{d} t \mathrm{~d} x \\
& =\int_{\mathbb{R}^{d}} \int_{\mathbb{R}} \varphi(t) \psi(x) \sigma(X(t, x))[\Delta u(X(t, x))-\Delta u(x)] \mathrm{d} t \mathrm{~d} x .
\end{aligned}
$$

Taking $\varphi(t)=\rho_{n}(t)$ in (2.32) and applying the limit (2.20) of Lemma 2.4 with $F=\sigma, \sigma, \sigma \Delta u$ in $L_{\text {loc }}^{p}\left(\mathbb{R}^{d}\right)$ for $p:=\frac{d}{d-1}$, and respectively $G=\nabla u \cdot \nabla \psi, \psi \Delta u, \psi$ in $L^{p^{\prime}}\left(\mathbb{R}^{d}\right)$ with compact support, we obtain that

$$
\int_{\mathbb{R}^{d}} \sigma(x) \nabla u(x) \cdot \nabla \psi(x) \mathrm{d} x=0, \quad \forall \psi \in C_{c}^{\infty}\left(\mathbb{R}^{d}\right)
$$

or equivalently the conductivity equation (2.3).

Proof of Lemma 2.4 .

(i) Let $I$ be a bounded interval of $\mathbb{R}$ and let $K$ be a compact set of $\mathbb{R}^{d}$. We have for any $t \in I$ and $x \in K$,

$$
\left|X_{n}(t, x)\right| \leq|x|+\left|\int_{0}^{t}\right| \nabla u_{n}\left(X_{n}(s, x)\right)|\mathrm{d} s| .
$$

Moreover, the uniform continuity of $\nabla u$ in $\mathbb{R}^{d}$ and the equality $\nabla u_{n}=\rho_{n} * \nabla u$ imply the existence of a constant $c>0$ such that

$$
\left|\nabla u_{n}(y)\right| \leq c|y|+c, \quad \forall n \in \mathbb{N}, \forall y \in \mathbb{R}^{d}
$$


We thus deduce that

$$
\left|X_{n}(t, x)\right| \leq c+c\left|\int_{0}^{t}\right| X_{n}(s, x)|\mathrm{d} s|, \quad \forall n \in \mathbb{N}, \forall t \in I, \forall x \in K .
$$

Hence, by Gronwall's inequality (see, e.g., [11], Sect. 17.3) there exists a constant $c>0$ such that

$$
\left|X_{n}(t, x)\right| \leq c e^{c|t|}, \quad \forall n \in \mathbb{N}, \forall t \in I, \forall x \in K
$$

Therefore, there exists a compact $\hat{K}$ of $\mathbb{R}^{d}$ and $E$, a set of Lebesgue measure zero, such that

$$
X_{n}(t, x), X(t, x) \in \hat{K}, \quad \forall n \in \mathbb{N}, \forall t \in I, \forall x \in K \backslash E .
$$

Let $f \in L_{\text {loc }}^{1}\left(\mathbb{R}^{d}\right)$, and let $f_{n}$ be a sequence in $C_{c}^{\infty}\left(\mathbb{R}^{d}\right)$ which converges strongly to $f$ in $L_{\text {loc }}^{1}\left(\mathbb{R}^{d}\right)$. We will show that $f_{n} \circ X$ converges strongly to some function $g$ in $L^{1}(I \times K)$. By Theorem II.3.1 from [9] and Theorem III.2 from [9] $f_{n} \circ X$ is in $L_{\text {loc }}^{1}\left(\mathbb{R} \times \mathbb{R}^{d}\right)$. Let $O$ be a bounded open set of $\mathbb{R}^{d}$ containing the compact set $\hat{K}$, and let $\psi$ be a non-negative function in $C_{c}(O)$ which is equal to 1 in $\hat{K}$. By (2.34) and estimate (2.17) we have for any $p, q \in \mathbb{N}$,

$$
\begin{aligned}
\int_{I} \int_{K}\left|f_{p}(X(t, x))-f_{q}(X(t, x))\right| \mathrm{d} t \mathrm{~d} x & \leq \int_{I} \mathrm{~d} t \int_{\mathbb{R}^{d}} \psi(X(t, x))\left|f_{p}(X(t, x))-f_{q}(X(t, x))\right| \mathrm{d} x \\
& =\int_{I} \mathrm{~d} t \int_{\mathbb{R}^{d}} \psi\left|f_{p}-f_{q}\right| \mathrm{d} \lambda_{X}(t) \leq c \int_{O}\left|f_{p}-f_{q}\right| .
\end{aligned}
$$

Hence, $f_{n} \circ X$ is a Cauchy sequence in $L^{1}(I \times K)$ and thus converges strongly to some function $g$ in $L^{1}(I \times$ $K)$. Therefore, due to the arbitrariness of $I, K$ the sequence $f_{n} \circ X$ converges strongly to some function $g$ in $L_{\text {loc }}^{1}\left(\mathbb{R} \times \mathbb{R}^{d}\right)$.

Finally, by estimate (2.16) we have for any bounded interval $I$ of $\mathbb{R}$, any bounded open set $O$ of $\mathbb{R}^{d}$ and any function $\varphi \in C_{c}(O)$,

$$
\begin{aligned}
& \int_{I} \mathrm{~d} t \int_{\mathbb{R}^{d}} \varphi f \mathrm{~d} \lambda_{X}(t)=\int_{I} \mathrm{~d} t \int_{O} \varphi(x) f(x) r(t, x) \mathrm{d} x=\lim _{n \rightarrow \infty} \int_{I} \mathrm{~d} t \int_{O} \varphi(x) f_{n}(x) r(t, x) \mathrm{d} x \\
& =\lim _{n \rightarrow \infty} \int_{I} \mathrm{~d} t \int_{\mathbb{R}^{d}} \varphi f_{n} \mathrm{~d} \lambda_{X}(t)=\lim _{n \rightarrow \infty} \int_{I} \int_{\mathbb{R}^{d}}\left(\varphi f_{n}\right)(X(t, x)) \mathrm{d} x=\int_{I} \mathrm{~d} t \int_{\mathbb{R}^{d}} \varphi(X(t, x)) g(t, x) \mathrm{d} x,
\end{aligned}
$$

which, due to the arbitrariness of $I, O, \varphi$, implies that $f \circ X=g \in L_{\mathrm{loc}}^{1}\left(\mathbb{R} \times \mathbb{R}^{d}\right)$.

(ii) Let $I$ be a bounded interval of $\mathbb{R}$ and let $K$ be a compact set of $\mathbb{R}^{d}$. Let $\varphi \in C_{c}^{\infty}\left(\mathbb{R}^{d}\right)$ be an approximation of $f$ in $L^{1}\left(\mathbb{R}^{d}\right)$. We have

$$
\begin{aligned}
& \limsup _{n \rightarrow \infty} \int_{K} \int_{I}\left|f\left(X_{n}(s, x)\right)-f(X(s, x))\right| \mathrm{d} s \mathrm{~d} x \\
& \leq \limsup _{n \rightarrow \infty} \int_{K} \int_{I}\left|\varphi\left(X_{n}(s, x)\right)-\varphi(X(s, x))\right| \mathrm{d} s \mathrm{~d} x \\
& +\limsup _{n \rightarrow \infty} \int_{K} \int_{I}|f-\varphi|\left(X_{n}(s, x)\right) \mathrm{d} s \mathrm{~d} x+\int_{K} \int_{I}|f-\varphi|(X(s, x)) \mathrm{d} s \mathrm{~d} x .
\end{aligned}
$$


On the one hand, the uniform convergence of $X_{n}(\cdot, x)$ to $X(\cdot, x)$ in $I$ combined with the continuity of $\varphi$ yields that

$$
\int_{I}\left|\varphi\left(X_{n}(s, x)\right)-\varphi(X(s, x))\right| \mathrm{d} s \underset{n \rightarrow \infty}{\longrightarrow} 0 \quad \text { a.e. } x \in K
$$

and estimate (2.33) combined with the continuity of $\varphi$ gives that

$$
\int_{I}\left|\varphi\left(X_{n}(s, x)\right)-\varphi(X(s, x))\right| \mathrm{d} s \leq c \quad \text { a.e. } x \in K
$$

Hence, by the Lebesgue dominated convergence theorem

$$
\lim _{n \rightarrow \infty} \int_{K} \int_{I}\left|\varphi\left(X_{n}(s, x)\right)-\varphi(X(s, x))\right| \mathrm{d} s \mathrm{~d} x=0 .
$$

Then, since by (2.34) there exists a set $E$, of Lebesgue measure zero, such that

$$
1_{K}(x) \leq \min \left(1_{\hat{K}}(X(t, x)), 1_{\hat{K}}\left(X_{n}(t, x)\right)\right), \quad \forall n \in \mathbb{N}, \forall t \in I, \forall x \in \mathbb{R}^{d} \backslash E,
$$

using the estimate (2.17) satisfied by the image measure $\lambda_{X}(s)$ with $\Delta u$ and the similar one satisfied by $\lambda_{X_{n}}(s)$ with $\Delta u_{n}$, we get that

$$
\begin{aligned}
& \limsup _{n \rightarrow \infty} \int_{K} \int_{I}|f-\varphi|\left(X_{n}(s, x)\right) \mathrm{d} s \mathrm{~d} x+\int_{K} \int_{I}|f-\varphi|(X(s, x)) \mathrm{d} s \mathrm{~d} x \\
& \leq \limsup _{n \rightarrow \infty} \int_{I} \int_{\mathbb{R}^{d}}\left(1_{\hat{K}}|f-\varphi|\right)\left(X_{n}(s, x)\right) \mathrm{d} x \mathrm{~d} s+\int_{I} \int_{\mathbb{R}^{d}}\left(1_{\hat{K}}|f-\varphi|\right)(X(s, x)) \mathrm{d} x \mathrm{~d} s \\
& =\limsup _{n \rightarrow \infty} \int_{I} \int_{\mathbb{R}^{d}} 1_{\hat{K}}(y)|f-\varphi|(y) \lambda_{X_{n}}(s)(\mathrm{d} y) \mathrm{d} s+\int_{I} \int_{\mathbb{R}^{d}} 1_{\hat{K}}(y)|f-\varphi|(y) \lambda_{X}(s)(\mathrm{d} y) \mathrm{d} s \\
& \leq c\|f-\varphi\|_{L^{1}(\hat{K})} .
\end{aligned}
$$

Therefore, putting this and limit (2.36) in (2.35) we deduce the desired limit (2.18).

(iii) Let $I$ be a bounded interval of $\mathbb{R}$, let $K$ be a compact set of $\mathbb{R}^{d}$, and let $\hat{K}$ be a compact set of $\mathbb{R}^{d}$ satisfying (2.34). Let $f_{n}$ be a non-negative sequence of $L_{\text {loc }}^{1}\left(\mathbb{R}^{d}\right)$ which converges strongly to 0 in $L_{\text {loc }}^{1}\left(\mathbb{R}^{d}\right)$. Repeating the argument of $(i i)$ using inequality (2.37) and the estimate (2.17) with $X_{n}$ in place of $X$, we get that

$$
\begin{aligned}
\limsup _{n \rightarrow \infty} \int_{K} \int_{I} f_{n}\left(X_{n}(s, x)\right) \mathrm{d} s \mathrm{~d} x & \leq \limsup _{n \rightarrow \infty} \int_{I} \int_{\mathbb{R}^{d}}\left(1_{\hat{K}} f_{n}\right)\left(X_{n}(s, x)\right) \mathrm{d} s \mathrm{~d} x \\
& \leq \limsup _{n \rightarrow \infty} \int_{I} \int_{\mathbb{R}^{d}} 1_{\hat{K}}(y) f_{n}(y) \lambda_{X_{n}}(s)(\mathrm{d} y) \mathrm{d} s \\
& \leq c \limsup _{n \rightarrow \infty}\left\|f_{n}\right\|_{L^{1}(\hat{K})}=0,
\end{aligned}
$$

which yields (2.19).

(iv) Let $F \in L_{\mathrm{loc}}^{p}\left(\mathbb{R}^{d}\right)^{N}$ for $N \in \mathbb{N}, p \in[1, \infty)$, and let $G \in L^{p^{\prime}}\left(\mathbb{R}^{d}\right)^{N}$ whose support is included in a compact set $K$ of $\mathbb{R}^{d}$. Consider a compact set $\hat{K}$ of $\mathbb{R}^{d}$ satisfying (2.34) with $I=[-1,1]$ and $K$, i.e. there exists a set $E$, 
of Lebesgue measure zero, such that

$$
1_{\hat{K}}(X(t, x))=1, \quad \forall t \in[-1,1], \forall x \in K \backslash E .
$$

Let $\Phi \in C_{c}^{\infty}\left(\mathbb{R}^{d}\right)^{N}$ be an approximation of $F$ in $L^{p}(\hat{K})^{N}$. By (2.6) we have

$$
\begin{aligned}
& \int_{\mathbb{R}^{d}} \int_{\mathbb{R}} \rho_{n}(s) F(X(s, x)) \cdot G(x) \mathrm{d} s \mathrm{~d} x-\int_{\mathbb{R}^{d}} F(x) \cdot G(x) \mathrm{d} x \\
& =\int_{\mathbb{R}^{d}} \int_{\mathbb{R}} \rho_{n}(s)[\Phi(X(s, x)-\Phi(x))] \cdot G(x) \mathrm{d} s \\
& +\int_{\mathbb{R}^{d}} \int_{\mathbb{R}} \rho_{n}(s)\left[\left(1_{\hat{K}}(F-\Phi)\right)(X(s, x))-\left(1_{\hat{K}}(F-\Phi)\right)(x)\right] \cdot G(x) \mathrm{d} s \mathrm{~d} x .
\end{aligned}
$$

Then, by the Hölder inequality combined with estimate (2.16) we get that

$$
\begin{aligned}
& \limsup _{n \rightarrow \infty}\left|\int_{\mathbb{R}^{d}} \int_{\mathbb{R}} \rho_{n}(s) F(X(s, x)) \cdot G(x) \mathrm{d} s \mathrm{~d} x-\int_{\mathbb{R}^{d}} F(x) \cdot G(x) \mathrm{d} x\right| \\
& \leq \limsup _{n \rightarrow \infty}\left|\int_{\mathbb{R}^{d}} \int_{\mathbb{R}} \rho_{n}(s)[\Phi(X(s, x))-\Phi(x)] \cdot G(x) \mathrm{d} s \mathrm{~d} x\right|+c\|F-\Phi\|_{L^{p}(\hat{K})^{N}}\|G\|_{L^{p^{\prime}}(K)^{N}} .
\end{aligned}
$$

By the continuity of $\Phi$ we have

$$
\int_{\mathbb{R}} \rho_{n}(s)[\Phi(X(s, x))-\Phi(x)] \mathrm{d} s \underset{n \rightarrow \infty}{\longrightarrow} 0 \quad \text { a.e. } x \in \mathbb{R}^{d}
$$

Moreover, we have

$$
\left|\int_{\mathbb{R}} \rho_{n}(s)[\Phi(X(s, x))-\Phi(x)] \mathrm{d} s\right| \leq 2\|\Phi\|_{L^{\infty}\left(\mathbb{R}^{d}\right)^{N}} \quad \text { a.e. } x \in \mathbb{R}^{d}
$$

so that

$$
\left|\left(\int_{\mathbb{R}} \rho_{n}(s)[\Phi(X(s, x))-\Phi(x)] \mathrm{d} s\right) \cdot G(x)\right| \leq c|G(x)| \text { a.e. } x \in \mathbb{R}^{d} .
$$

Hence, since $G \in L^{1}\left(\mathbb{R}^{d}\right)^{N}$ due to its compact support, the Lebesgue dominated convergence theorem implies that

$$
\lim _{n \rightarrow \infty} \int_{\mathbb{R}^{d}} \int_{\mathbb{R}} \rho_{n}(s)[\Phi(X(s, x))-\Phi(x)] \cdot G(x) \mathrm{d} s \mathrm{~d} x=0 .
$$

Using this in (2.38) we thus obtain limit (2.20).

\section{CASE WhERE THE GRADIENT FIELD HAS JUMPS}

In this section we will consider a gradient field which is piecewise regular in a finite number of so-called gradient-admissible domains. 


\subsection{Gradient-admissible domain}

The starting point is the following result first due to Bongiorno, Valente [4], and well reformulated by Richter $[16]$.

Proposition 3.1 ([16], Lem. 2). Let $\Omega$ be a bounded domain (i.e. a connected open set) of $\mathbb{R}^{d}$, and let $u \in C^{2}(\bar{\Omega})$ such that

$$
\inf _{\Omega}|\nabla u|>0
$$

Let $\Gamma_{-}$be the inflow boundary of $\Omega$, i.e. the subset of $\partial \Omega$ on which the outer normal derivative of $u$ is negative: $\frac{\partial u}{\partial \nu}<0$, and let $\Gamma_{+}$be the outflow boundary of $\Omega$, i.e. the subset of $\partial \Omega$ on which the outer normal derivative of $u$ is positive: $\frac{\partial u}{\partial \nu}>0$.

Then, each point of $\Omega$ belongs to a unique trajectory $t \mapsto X(t, x)$ which flows from $\Gamma_{-}$to $\Gamma_{+}$. Moreover, there exists a unique positive function $\sigma \in C^{1}(\bar{\Omega})$ taking prescribed values on $\Gamma_{-}$(resp. on $\left.\Gamma_{+}\right)$which is solution to the equation $\operatorname{div}(\sigma \nabla u)=0$ in $\Omega$.

Remark 3.2. Actually, in [16] the existence and the uniqueness of the conductivity $\sigma$ taking previous values on the inflow boundary $\Gamma_{-}$is proved under the weaker assumption

$$
\inf _{\Omega}(\min (|\nabla u|, \Delta u))>0
$$

However, we will need the stronger condition (3.1) in the sequel.

Proof of Proposition 3.1. The proof can be found in [16]. We will give another expression of the conductivity $\sigma$ following Theorem 2.1. Let $\gamma$ be a positive function in $C^{1}\left(\overline{\Gamma_{-}}\right)$. For a fixed $x \in \Omega$, the trajectory $t \in\left[\tau_{-}(x), \tau_{+}(x)\right] \mapsto X(t, x)$ flows from the inflow boundary $\Gamma_{-}$to the outflow boundary $\Gamma_{+}$, where $\tau_{-}(x)<$ $0<\tau_{+}(x)$ and $X\left(\tau_{ \pm}(x), x\right) \in \Gamma_{ \pm}$. Let $y=X(\tau, x)$ be a point on the same trajectory. Note that by the semi-group property of the flow we have

$$
X\left(\tau_{-}(x), x\right)=X\left(\tau_{-}(y), y\right)=X\left(\tau_{-}(y), X(\tau, x)\right)=X\left(\tau_{-}(y)+\tau, x\right)
$$

hence $\tau_{-}(y)=\tau_{-}(x)-\tau$. Now, we can define the conductivity $\sigma_{\gamma}$ along the trajectory by

$$
\sigma_{\gamma}(X(t, x)):=\gamma\left(X\left(\tau_{-}(x), x\right)\right) \exp \left(\int_{t}^{\tau_{-}(x)} \Delta u(X(s, x)) \mathrm{d} s\right) \quad \text { for } t \in\left[\tau_{-}(x), \tau_{+}(x)\right]
$$

Formula (3.2) does not depend on the point $y=X(\tau, x)$ on the same trajectory, since

$$
\int_{\tau_{-}(y)}^{t} \Delta u(X(s, y)) \mathrm{d} s=\int_{\tau_{-}(x)-\tau}^{t} \Delta u(X(s+\tau, x)) \mathrm{d} s=\int_{\tau_{-}(x)}^{t+\tau} \Delta u(X(s, x)) \mathrm{d} s,
$$

which implies that $\sigma_{\gamma}(X(t, y))=\sigma_{\gamma}(X(t+\tau, x))$. Moreover, it is immediate that formula (3.2) implies formula (2.5). Therefore, by Theorem $2.1 \sigma_{\gamma}$ is a solution to the equation $\operatorname{div}\left(\sigma_{\gamma} \nabla u\right)=0$ in $\Omega$, and $\sigma_{\gamma}=\gamma$ on $\Gamma_{-}$.

Conversely, consider a positive function $\sigma \in C^{1}(\bar{\Omega})$ such that $\operatorname{div}(\sigma \nabla u)=0$ in $\Omega$, and $\sigma=\gamma$ on $\Gamma_{-}$. From the equality $\nabla \sigma \cdot \nabla u+\sigma \Delta u=0$ in $\Omega$, we deduce that for any $x \in \Omega$,

$$
\frac{\mathrm{d}}{\mathrm{d} t}\left[\ln (\sigma(X(t, x))]=-\Delta u(X(t, x)), \quad \forall t \in\left[\tau_{-}(x), \tau_{+}(x)\right]\right.
$$




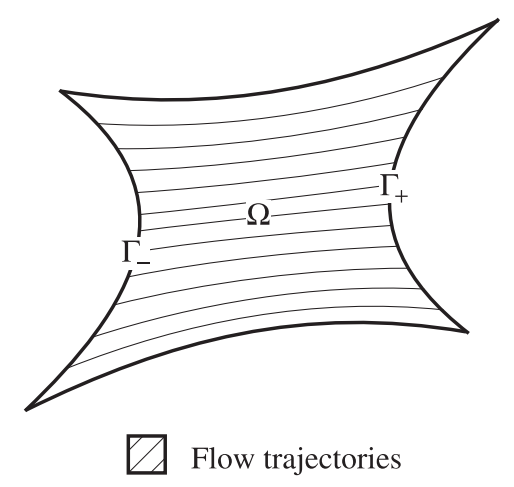

Figure 1. The trajectories in $\Omega$ flow from $\Gamma_{-}$to $\Gamma_{+}$.

then

$$
\frac{\sigma(x)}{\sigma(X(t, x))}=\exp \left(\int_{0}^{t} \Delta u(X(s, x)) \mathrm{d} s\right), \quad \forall t \in\left[\tau_{-}(x), \tau_{+}(x)\right]
$$

This combined with (3.2) implies that for any $x \in \Omega$,

$$
\frac{\sigma(x)}{\gamma\left(X\left(\tau_{-}(x), x\right)\right)}=\frac{\sigma(x)}{\sigma\left(X\left(\tau_{-}(x), x\right)\right)}=\exp \left(\int_{0}^{\tau_{-}(x)} \Delta u(X(s, x)) \mathrm{d} s\right)=\frac{\sigma_{\gamma}(x)}{\gamma\left(X\left(\tau_{-}(x), x\right)\right)}
$$

Therefore, we obtain that $\sigma=\sigma_{\gamma}$ in $\Omega$, which shows the uniqueness of the conductivity $\sigma_{\gamma}$.

We can now state the definition of a gradient-admissible set.

Definition 3.3. Let $\Omega$ be a bounded domain of $\mathbb{R}^{d}$, and let $u \in C^{2}(\bar{\Omega})$. The domain $\Omega$ is said to be $\nabla u$-admissible if condition (3.1) holds.

Remark 3.4. The boundary of a $\nabla u$-admissible domain $\Omega$ is split into the inflow boundary $\Gamma_{-}$, the outflow boundary $\Gamma_{+}$, and surfaces which are tangential to $\nabla u$. Figure 1 shows a two-dimensional $\nabla u$-admissible domain $\Omega$ with two boundary curves which are tangential to $\nabla u$.

\subsection{Piecewise regular gradient field}

In connection with Definition 3.3 of a gradient-admissible set, we focus on a so-called admissible domain defined as follows.

Definition 3.5. Let $\Omega$ be a bounded domain of $\mathbb{R}^{d}$. The set $\Omega$ is said to be admissible if it is decomposed into "generalized open polyhedra" (obtained from polyhedra through a smooth diffeomorphism) $\Omega_{j, k}$ for $j \in$ $\left\{1, \ldots, n_{k}\right\}$ and $k \in\{1, \ldots, n\}$, where some of the domains $\Omega_{1, k}$ may agree, satisfying:

( $i$ ) each polyhedron $\Omega_{j, k}$ is a $\nabla u_{j, k}$-admissible domain with $u_{j, k} \in C^{2}\left(\overline{\Omega_{j, k}}\right)$;

(ii) each internal face of the chain $\Omega_{1, k} \rightarrow \Omega_{2, k} \rightarrow \cdots \rightarrow \Omega_{n_{k}, k}$ made of $n_{k}$ contiguous domains, is an inflow boundary for one domain and an outflow boundary for the contiguous domain, or equivalently

$$
\frac{\partial u_{j, k}}{\partial \nu} \frac{\partial u_{j-1, k}}{\partial \nu}>0 \quad \text { on } \partial \Omega_{j, k} \cap \partial \Omega_{j-1, k} \quad \text { for any } j \in\left\{2, \ldots, n_{k}\right\}
$$

where $\nu$ is the outer normal of $\partial \Omega_{j, k}$; 

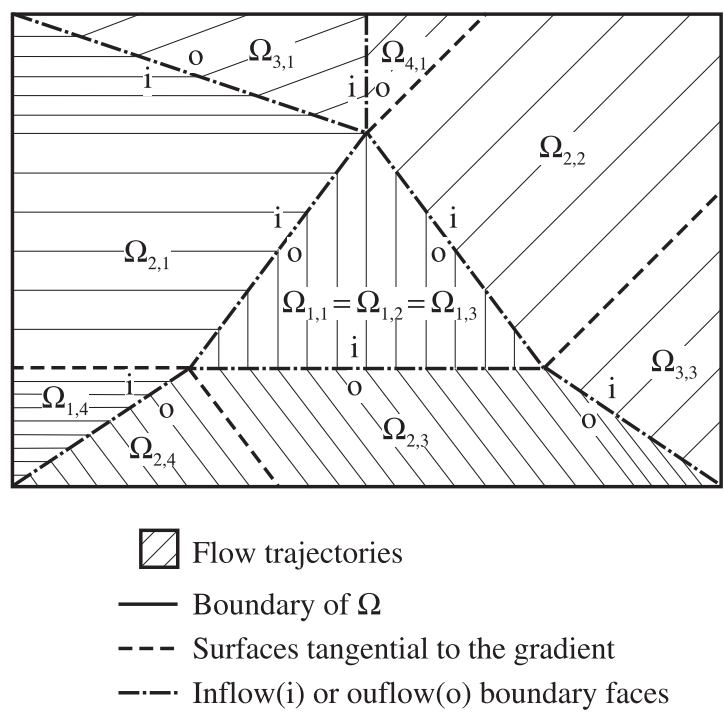

Figure 2. An admissible domain $\Omega$ composed of $n=4$ chains.

(iii) each external face of the chain $\Omega_{1, k} \rightarrow \Omega_{2, k} \rightarrow \cdots \rightarrow \Omega_{n_{k}, k}$ is

- either a boundary part of $\partial \Omega$,

$\circ$ or a surface tangential to some $\nabla u_{j, k}$,

o or an inflow or outflow boundary of $\Omega_{1, k}$ which is (possibly) connected to another chain $\Omega_{1, k}=\Omega_{1, j} \rightarrow$ $\Omega_{2, j} \rightarrow \cdots \rightarrow \Omega_{n_{j}, j}$

\section{Example 3.6.}

1. Figure 2 represents an admissible domain $\Omega$ composed of the $n=4$ chains

$$
\left\{\begin{array}{l}
\Omega_{1,1} \rightarrow \Omega_{2,1} \rightarrow \Omega_{3,1} \rightarrow \Omega_{4,1} \\
\Omega_{1,1}=\Omega_{1,2} \rightarrow \Omega_{2,2} \\
\Omega_{1,1}=\Omega_{1,3} \rightarrow \Omega_{2,3} \rightarrow \Omega_{3,3} \\
\Omega_{1,4} \rightarrow \Omega_{2,4}
\end{array}\right.
$$

The three first chains are connected to the same set $\Omega_{1,1}$. The fourth one is separated from three others by surfaces which are tangential to the gradient.

2. The domain $\Omega$ of Figure 3 is composed of $n=1$ chain made of $4 \nabla u_{k}$-admissible sets. It is not admissible, since the chain $\Omega_{1} \rightarrow \Omega_{2} \rightarrow \Omega_{3} \rightarrow \Omega_{4}$ has an external boundary which is neither a boundary part of $\partial \Omega$ nor a surface tangential to some gradient $\nabla u_{k}$. This creates a conflict for defining a suitable conductivity $\sigma_{k}$ in each domain $\Omega_{k}$ (see Rem. 3.8, 2).

Theorem 3.7. Let $\Omega$ be an admissible domain composed of $\nabla u_{j, k}$-admissible open sets $\Omega_{j, k}$ for $j \in\left\{1, \ldots, n_{k}\right\}$ and $k \in\{1, \ldots, n\}$, according to Definition 3.5, and let $u \in C(\bar{\Omega})$ be such that $u=u_{j, k}$ in $\overline{\Omega_{j, k}}$. Then, there exists a piecewise continuous positive conductivity $\sigma$ such that

$$
\begin{cases}\sigma_{\mid \overline{\Omega_{k, j}}} \in C^{1}\left(\overline{\Omega_{k, j}}\right) & \text { for } j \in\left\{1, \ldots, n_{k}\right\} \text { and } k \in\{1, \ldots, n\}, \\ \operatorname{div}(\sigma \nabla u)=0 & \text { in } \mathscr{D}^{\prime}(\Omega) .\end{cases}
$$



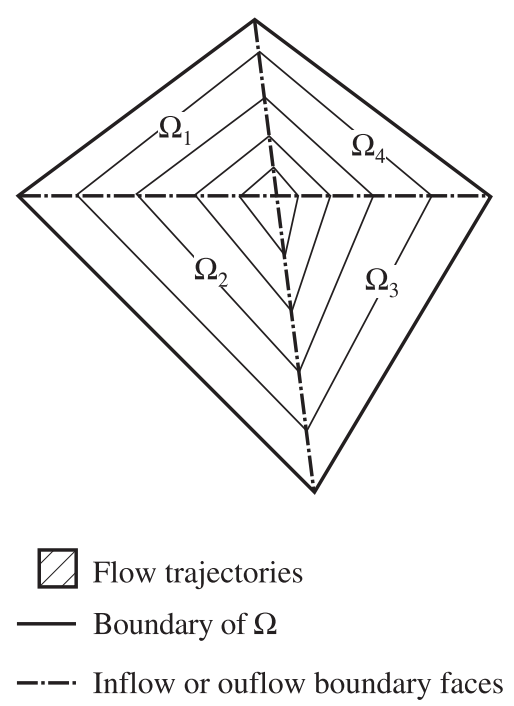

FiguRE 3 . A non-admissible domain $\Omega$ with $n=1$ chain: $\Omega_{1} \rightarrow \Omega_{2} \rightarrow \Omega_{3} \rightarrow \Omega_{4}$.

Conversely, let $\Omega$ be a bounded domain of $\mathbb{R}^{d}$ composed of $n$ generalized polyhedra $\Omega_{k}$, and let $u$ be a function in $C(\bar{\Omega})$ such that $u_{k}:=u_{\mid \overline{\Omega_{k}}} \in C^{2}\left(\overline{\Omega_{k}}\right)$ and $\Omega_{k}$ is a $\nabla u_{k}$-admissible domain for $k \in\{1, \ldots, n\}$. Assume that $\sigma$ is a positive function in $C(\bar{\Omega})$ such that $\sigma_{k}:=\sigma_{\mid \overline{\Omega_{k}}} \in C^{1}\left(\overline{\Omega_{k}}\right)$ and $\operatorname{div}(\sigma \nabla u)=0$ in $\mathscr{D}^{\prime}(\Omega)$. Then, for any contiguous polyhedra $\Omega_{j}$ and $\Omega_{k}$, the common face $\Gamma_{j, k}:=\partial \Omega_{j} \cap \partial \Omega_{k}$ is either a surface tangential to $\nabla u$, or an inflow (resp. outflow) boundary of $\Omega_{j}$ and an outflow (resp. inflow) boundary of $\Omega_{k}$.

Proof of Theorem 3.7. The idea is to construct in each chain $\Omega_{1, k} \rightarrow \Omega_{2, k} \rightarrow \cdots \rightarrow \Omega_{n_{k}, k}$ for $k \in\{1, \ldots, n\}$, successively the conductivities $\sigma_{1, k}, \ldots, \sigma_{n_{k}, k}$. To this end, the conductivity $\sigma_{j-1, k}$ being constructed in the domain $\Omega_{j-1, k}$ for some $j \in\left\{2, \ldots, n_{k}\right\}$, we will choose a suitable positive continuous function $\gamma_{j, k}$ on the inflow or outflow boundary face $\partial \Omega_{j, k} \cap \partial \Omega_{j-1, k}$, which

- determines the conductivity $\sigma_{j, k}$ in the $\nabla u_{j, k}$-admissible domain $\Omega_{j, k}$ by Proposition 3.1,

- satisfies the flux continuity condition through the surface $\partial \Omega_{j, k} \cap \partial \Omega_{j-1, k}$.

For $k \in\{1, \ldots, n\}$, fix the conductivity equal to 1 on the inflow or outflow boundary face of $\Omega_{1, k}$, which by Proposition 3.1 determines a unique conductivity $\sigma_{1, k} \in C^{1}\left(\overline{\Omega_{1, k}}\right)$ such that $\operatorname{div}\left(\sigma_{1, k} \nabla u\right)=0$ in $\Omega_{1, k}$.

Next, using an induction argument we will construct a suitable piecewise continuous conductivity along the chain $\Omega_{1, k} \rightarrow \cdots \rightarrow \Omega_{n_{k}, k}$. Assume that for some $j \in\left\{2, \ldots, n_{k}\right\}$, we have built a piecewise conductivity $\sigma=\sigma_{i, k}$ in $\overline{\Omega_{i, k}}$ for $i \in\{1, \ldots, j-1\}$, solution to the equation

$$
\operatorname{div}(\sigma \nabla u)=0 \text { in int }\left[\Omega_{1, k} \cup \cup_{i=2}^{j-1}\left(\Omega_{i, k} \cup \Gamma_{i, k}\right)\right],
$$

where $\Gamma_{i, k}:=\partial \Omega_{i, k} \cap \partial \Omega_{i-1, k}$ is the common face of $\Omega_{j, k}$ and $\Omega_{j-1, k}$. By the condition (3.3) on $\Gamma_{j, k}$ there exists a positive function $\gamma_{j, k} \in C\left(\Gamma_{j, k}\right)$ such that

$$
\gamma_{j, k} \frac{\partial u_{j, k}}{\partial \nu}=\sigma_{j-1, k} \frac{\partial u_{j-1, k}}{\partial \nu} \quad \text { on } \Gamma_{j, k}
$$

where $\nu$ is the outer normal of $\partial \Omega_{j, k}$. Since by the assumption (ii) of Definition $3.5 \Gamma_{j, k}$ is an inflow or outflow boundary face of the $\nabla u_{j, k}$-admissible domain $\Omega_{j, k}$, by Proposition 3.1 there exists a positive conductivity $\sigma_{j, k} \in C\left(\overline{\Omega_{j, k}}\right)$ taking the value $\gamma_{j, k}$ on $\Gamma_{j, k}$ and solution to the equation $\operatorname{div}\left(\sigma_{j, k} \nabla u\right)=0$ in $\Omega_{j, k}$. Then, 
equality (3.5) reads as the flux continuity condition through $\Gamma_{j, k}$. It follows that the conductivity $\sigma:=\sigma_{i, k}$ in $\overline{\Omega_{i, k}}$ for $i \in\{1, \ldots, j\}$, is solution to the equation

$$
\operatorname{div}(\sigma \nabla u)=0 \text { in int }\left[\Omega_{1, k} \cup \cup_{i=2}^{j}\left(\Omega_{i, k} \cup \Gamma_{i, k}\right)\right]
$$

which concludes the induction proof. Therefore, we has just constructed a piecewise continuous positive function

$$
\sigma=\sigma_{j, k} \text { in } \overline{\Omega_{j, k}} \quad \text { solution to } \operatorname{div}(\sigma \nabla u)=0 \text { in } \operatorname{int}\left[\Omega_{1, k} \cup \cup_{j=2}^{n_{k}}\left(\Omega_{j, k} \cup \Gamma_{j, k}\right)\right] \text {. }
$$

Now, according to Definition 3.5 consider the partition $\left(K_{i}\right)_{1 \leq i \leq p}$ of $\{1, \ldots, n\}$ such that the sets $\Omega_{1, k}$ agree to the same set $\Omega_{1, k_{i}}\left(k_{i} \in K_{i}\right)$ for any $k \in K_{i}$ and $i \in\{1, \ldots, p\}$. Since for each $i \in\{1, \ldots, p\}$ the chains $\Omega_{1, k} \rightarrow \Omega_{2, k} \rightarrow \cdots \rightarrow \Omega_{n_{k}, k}$ are connected to the set $\Omega_{1, k_{i}}$ for any $k \in K_{i}$, by the definition (3.6) of the piecewise continuous conductivity $\sigma$ we thus have

$$
\operatorname{div}(\sigma \nabla u)=0 \quad \text { in } \operatorname{int}\left(\bigcup_{k \in K_{i}}\left[\Omega_{1, k_{i}} \cup \cup_{j=2}^{n_{k}}\left(\Omega_{j, k} \cup \Gamma_{j, k}\right)\right]\right) \quad \text { for any } i \in\{1, \ldots, p\} \text {. }
$$

Moreover, by the assumption (iii) of Definition 3.5 we have

$$
\frac{\partial u}{\partial \nu}=0 \quad \text { on } \partial\left(\bigcup_{k \in K_{i}}\left[\Omega_{1, k_{i}} \cup \cup_{j=2}^{n_{k}}\left(\Omega_{j, k} \cup \Gamma_{j, k}\right)\right]\right) \backslash \partial \Omega \quad \text { for any } i \in\{1, \ldots, p\} \text {. }
$$

Let $\varphi \in C_{c}^{\infty}(\Omega)$. Therefore, integrating by parts and using (3.7), (3.8) we get that

$$
\int_{\Omega} \sigma \nabla u \cdot \nabla \varphi \mathrm{d} x=\sum_{i=1}^{p} \int_{\bigcup_{k \in K_{i}}\left[\Omega_{1, k_{i}} \cup \cup_{j=2}^{n_{k}}\left(\Omega_{j, k} \cup \Gamma_{j, k}\right)\right]} \sigma \nabla u \cdot \nabla \varphi \mathrm{d} x=0,
$$

which implies that the piecewise continuous conductivity $\sigma$ of (3.6) is solution to the equation $\operatorname{div}(\sigma \nabla u)=0$ in $\mathscr{D}^{\prime}(\Omega)$.

Conversely, let $\Omega$ be a bounded domain of $\mathbb{R}^{d}$ composed of $n$ generalized polyhedra $\Omega_{k}$ for $k \in\{1, \ldots, n\}$. Let $u \in C(\bar{\Omega})$ be such that $u_{k}:=u_{\mid \overline{\Omega_{k}}} \in C^{2}\left(\overline{\Omega_{k}}\right)$, and $\Omega_{k}$ is $\nabla u_{k}$-admissible. Assume that $\sigma$ is a positive piecewise continuous function such that $\sigma_{k}:=\sigma_{\mid \overline{\Omega_{k}}} \in C^{1}\left(\overline{\Omega_{k}}\right)$ and $\operatorname{div}(\sigma \nabla u)=0$ in $\mathscr{D}^{\prime}(\Omega)$. Consider two contiguous polyhedra $\Omega_{j}$ and $\Omega_{k}$, the common face of which $\Gamma_{j, k}:=\partial \Omega_{j} \cap \partial \Omega_{k}$ is not a surface tangential to $\nabla u$. The flux continuity condition through $\Gamma_{j, k}$ reads as

$$
\sigma_{j} \frac{\partial u_{j}}{\partial \nu}=\sigma_{k} \frac{\partial u_{k}}{\partial \nu} \quad \text { on } \Gamma_{j, k}
$$

where $\nu$ is the outer normal to $\partial \Omega_{j}$, which implies that

$$
\frac{\partial u_{j}}{\partial \nu} \frac{\partial u_{k}}{\partial \nu}>0 \text { on } \Gamma_{j, k}
$$

Therefore, $\Gamma_{j, k}$ is an inflow (resp. outflow) boundary face of $\Omega_{j}$, and an outflow (resp. inflow) boundary face of $\Omega_{k}$. The proof of Theorem 3.7 is now complete. 


\section{Remark 3.8.}

1. In the case of Figure 2 the domain $\Omega$ is composed of 9 polyhedra $\Omega_{j, k}$ grouped into 4 chains with 11 internal faces. The step by step construction of Theorem 3.7 reads as follows:

- We prescribe the conductivity on the say inflow face $\partial \Omega_{1,1} \cap \partial \Omega_{2,3}$ of $\Omega_{1,1}$, which determines the conductivity $\sigma_{1,1}$. Then, $\partial \Omega_{1,1} \cap \partial \Omega_{2,1}$ and $\partial \Omega_{1,1} \cap \partial \Omega_{2,2}$ are outflow faces of $\Omega_{1,1}$.

- We choose successively the conductivities on the inflow face $\partial \Omega_{1,1} \cap \partial \Omega_{2,1}$ of $\Omega_{2,1}$, the outflow face $\partial \Omega_{2,1} \cap \partial \Omega_{3,1}$ of $\Omega_{3,1}$, and the outflow face $\partial \Omega_{3,1} \cap \partial \Omega_{4,1}$ of $\Omega_{4,1}$, which determine the conductivities $\sigma_{2,1}, \sigma_{3,1}, \sigma_{4,1}$ ensuring the flux continuity conditions on $\partial \Omega_{1,1} \cap \partial \Omega_{2,1}, \partial \Omega_{2,1} \cap \partial \Omega_{3,1}, \partial \Omega_{3,1} \cap \partial \Omega_{4,1}$.

- We choose the conductivity on the inflow face $\partial \Omega_{1,1} \cap \partial \Omega_{2,2}$ of $\Omega_{2,2}$, which determines the conductivity $\sigma_{2,2}$ ensuring the flux continuity condition on $\partial \Omega_{1,1} \cap \partial \Omega_{2,2}$.

- We choose successively the conductivities on the outflow face $\partial \Omega_{1,1} \cap \partial \Omega_{2,3}$ of $\Omega_{2,3}$ and the inflow face $\partial \Omega_{2,3} \cap \partial \Omega_{3,3}$ of $\Omega_{3,3}$, which determine the conductivities $\sigma_{2,3}, \sigma_{3,3}$ ensuring the flux continuity conditions on $\partial \Omega_{1,1} \cap \partial \Omega_{2,3}, \partial \Omega_{2,3} \cap \partial \Omega_{3,3}$.

- We prescribe the conductivity on the say inflow face $\partial \Omega_{1,4} \cap \partial \Omega_{2,4}$ of $\Omega_{1,4}$, which determines the conductivity $\sigma_{1,4}$. Then, we choose the conductivity on the ouflow face $\partial \Omega_{1,4} \cap \partial \Omega_{2,4}$ of $\Omega_{2,4}$, which determines the conductivity $\sigma_{2,4}$ ensuring the flux continuity condition on $\partial \Omega_{1,4} \cap \partial \Omega_{2,4}$.

- The 4 remaining faces $\partial \Omega_{4,1} \cap \partial \Omega_{2,2}, \partial \Omega_{2,2} \cap \partial \Omega_{3,3}, \partial \Omega_{2,3} \cap \partial \Omega_{2,4}, \partial \Omega_{2,1} \cap \partial \Omega_{1,4}$ are tangential to the gradient, and thus satisfy the flux continuity conditions.

2. In the case of Figure 2 the domain $\Omega$ is made of one chain composed of 4 polyhedra. For example, we prescribe the conductivity on the say inflow face $\partial \Omega_{1} \cap \partial \Omega_{2}$ of $\Omega_{1}$. Then, the flux continuity conditions on the faces $\partial \Omega_{1} \cap \partial \Omega_{2}, \partial \Omega_{2} \cap \partial \Omega_{3}, \partial \Omega_{3} \cap \partial \Omega_{4}$ determine successively the conductivities $\sigma_{k}$ in $\Omega_{k}$ for $k=1,2,3,4$. But then the flux continuity condition on the face $\partial \Omega_{1} \cap \partial \Omega_{4}$ does not hold in general.

\section{EXAMPLES}

\subsection{Example 1}

Let $\Omega$ be an open set of $\mathbb{R}^{2}$ which is star-shaped with respect to the origin. Let $\xi_{1}, \ldots, \xi_{n}$ be $n \geq 2$ non-zero vectors of $\mathbb{R}^{2}$ such that the open cones

$$
\begin{cases}\Omega_{k}:=\left\{s \xi_{k}+t \xi_{k+1},, s, t>0\right\} & \text { for } 1 \leq k \leq n-1 \\ \Omega_{n}:=\left\{s \xi_{1}+t \xi_{n},, s, t>0\right\} & \text { for } k=n,\end{cases}
$$

do not contain any vector $\xi_{j}$.

Consider a function $u \in C(\bar{\Omega})$ of finite element type $\mathbb{P}_{1}$ (see, e.g. [8], Sect. 2.2), i.e. there exists constant vectors $\lambda_{k} \in \mathbb{R}^{2}$ such that

$$
\nabla u=\lambda_{k} \text { in } \Omega_{k} \quad \text { for } k \in\{1, \ldots, n\} .
$$

This imposes the flux continuity conditions

$$
\left(\lambda_{k}-\lambda_{k-1}\right) \cdot \xi_{k}=0, \quad \forall k \in\{2, \ldots, n\} \quad \text { and } \quad\left(\lambda_{1}-\lambda_{n}\right) \cdot \xi_{1}=0 .
$$

Up to decrease the value of $n$ we can also assume that

$$
\lambda_{k}-\lambda_{k-1} \neq 0, \quad \forall k \in\{2, \ldots, n\} \quad \text { and } \quad \lambda_{1}-\lambda_{n} \neq 0 .
$$

Similarly to the case of Figure 3 (see Rem. 3.8,2) the chain $\Omega_{1} \rightarrow \Omega_{2} \rightarrow \cdots \rightarrow \Omega_{n}$ does not satisfy the condition (iii) of Definition 3.5. Indeed, the existence of constant conductivities $\sigma_{k}$ in $\Omega_{k}$ satisfying the flux 


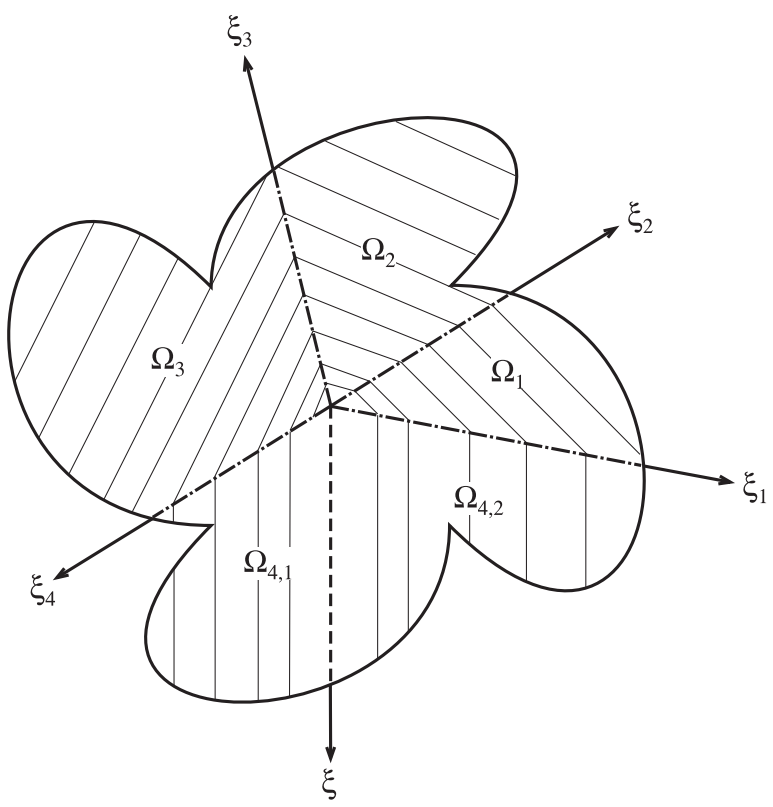

Flow trajectories

Boundary of $\Omega$

---. Surface tangential to the gradient

-.-. Inflow or ouflow boundary faces

FiguRE 4 . Triangulation of $\Omega$ by the cones $\Omega_{1}, \Omega_{2}, \Omega_{3}$, and $\Omega_{4}=\operatorname{int}\left(\overline{\Omega_{4,1}} \cup \overline{\Omega_{4,2}}\right)$ with $\xi \| \lambda_{4}$.

continuity condition $(3.9)$ reads as

$$
\sigma_{k} \operatorname{det}\left(\xi_{k}, \lambda_{k}\right)=\sigma_{k-1} \operatorname{det}\left(\xi_{k}, \lambda_{k-1}\right), \quad \forall k \in\{2, \ldots, n\} \quad \text { and } \quad \sigma_{n} \operatorname{det}\left(\xi_{1}, \lambda_{n}\right)=\sigma_{1} \operatorname{det}\left(\xi_{1}, \lambda_{1}\right),
$$

which thus implies the constraint

$$
\prod_{k=1}^{n} \operatorname{det}\left(\xi_{k}, \lambda_{k}\right)=\operatorname{det}\left(\xi_{1}, \lambda_{n}\right) \prod_{k=2}^{n} \operatorname{det}\left(\xi_{k}, \lambda_{k-1}\right) .
$$

A less restrictive alternative is to assume that for some $k \in\{1, \ldots, n\}$, say $k=n$ without loss of generality, there exists a vector $\xi \in \mathbb{R}^{2}$ satisfying

$$
\xi \in \Omega_{n} \backslash\{0\} \text { and } \xi \| \lambda_{n} .
$$

Hence, defining the subsets of $\Omega_{n}$

$$
\Omega_{n, 1}:=\left\{s \xi+t \xi_{n},, s, t>0\right\} \quad \text { and } \quad \Omega_{n, 2}:=\left\{s \xi+t \xi_{1},, s, t>0\right\},
$$

we have

$$
\frac{\partial u}{\partial \nu}=0 \quad \text { on } \partial \Omega_{n, 1} \cap \partial \Omega_{n, 2} \subset \mathbb{R} \xi
$$


Therefore, by (4.2) and (4.7) the chain $\Omega_{n, 2} \rightarrow \Omega_{1} \rightarrow \cdots \rightarrow \Omega_{n-1} \rightarrow \Omega_{n, 1}$ satisfies the conditions ( $i$ ) and (iii) of Definition 3.5 (see Fig. 4 and compare to Fig. 3). Then, taking into account conditions (4.3) and (4.4) the condition $(\mathrm{ii})$ of Definition 3.5 is equivalent to

$$
\operatorname{det}\left(\xi_{k}, \lambda_{k}\right) \operatorname{det}\left(\xi_{k}, \lambda_{k-1}\right)>0, \forall k \in\{2, \ldots, n\} \quad \text { and } \quad \operatorname{det}\left(\xi_{1}, \lambda_{1}\right) \operatorname{det}\left(\xi_{1}, \lambda_{n}\right)>0 .
$$

Therefore, by Theorem 3.7 $\nabla u$ is isotropically realizable in $\Omega$ if and only if condition (4.8) holds true. Finally, due to condition (4.8) a suitable piecewise constant conductivity is given by

$$
\sigma=\left\{\begin{array}{cl}
\frac{\operatorname{det}\left(\xi_{1}, \lambda_{n}\right)}{\operatorname{det}\left(\xi_{1}, \lambda_{1}\right)} & \text { in } \Omega_{1} \\
\frac{\operatorname{det}\left(\xi_{1}, \lambda_{n}\right)}{\operatorname{det}\left(\xi_{1}, \lambda_{1}\right)} \prod_{j=2}^{k} \frac{\operatorname{det}\left(\xi_{j}, \lambda_{j-1}\right)}{\operatorname{det}\left(\xi_{j}, \lambda_{j}\right)} & \text { in } \Omega_{k} \quad \text { for } 2 \leq k \leq n-1 \\
\frac{\operatorname{det}\left(\xi_{1}, \lambda_{n}\right)}{\operatorname{det}\left(\xi_{1}, \lambda_{1}\right)} \prod_{j=2}^{n} \frac{\operatorname{det}\left(\xi_{j}, \lambda_{j-1}\right)}{\operatorname{det}\left(\xi_{j}, \lambda_{j}\right)} & \text { in } \Omega_{n, 1} \\
1 & \text { in } \Omega_{n, 2} .
\end{array}\right.
$$

Remark 4.1. We can also extend the previous two-dimensional example to dimension three replacing the open cones (4.1) as follows. Let $\Omega$ be an open set of $\mathbb{R}^{3}$ which is star-shaped with respect to the origin. Let $\xi_{1}, \ldots, \xi_{n}$ be $n \geq 3$ non-zero vectors of $\mathbb{R}^{3}$ such that the open cones

$$
\Omega_{i, j, k}:=\Omega \cap\left\{r \xi_{i}+s \xi_{j}+t \xi_{k}, r, s, t>0\right\} \quad \text { if } \operatorname{det}\left(\xi_{i}, \xi_{j}, \xi_{k}\right) \neq 0,
$$

do not contain any vector $\xi_{\ell}$. For example, if $\left(e_{1}, e_{2}, e_{3}\right)$ is a basis of $\mathbb{R}^{3}$ and $n=6$ with

$$
\xi_{1}=e_{1}, \xi_{2}=e_{2}, \xi_{3}=e_{3}, \xi_{4}=-e_{1}, \xi_{5}=-e_{2}, \xi_{6}=-e_{3},
$$

there are 8 open cones of type (4.10).

\subsection{Example 2}

Let $f$ be a function in $W_{\text {loc }}^{2, \infty}\left(\mathbb{R}^{d-1}\right)$ for $d \geq 2$, and let $g, h$ be 2 functions in $C^{2}(\mathbb{R})$ such that

$$
\left\{\begin{array}{l}
f \text { satisfies condition }(2.2) \text { in } \mathbb{R}^{d-1}, \\
g(0)=h(0), \\
g^{\prime}, h^{\prime} \text { are uniformly continuous in } \mathbb{R} \text { and } g^{\prime}(t) h^{\prime}(t) \neq 0, \forall t \in \mathbb{R} .
\end{array}\right.
$$

Consider the function $u \in C\left(\mathbb{R}^{d}\right)$ defined by

$$
u(x)= \begin{cases}u_{1}\left(x_{1}, x^{\prime}\right):=g\left(x_{1}\right)+f\left(x^{\prime}\right) & \text { if }\left(x_{1}, x^{\prime}\right) \in \Omega_{1}:=(0, \infty) \times \mathbb{R} \\ u_{2}\left(x_{1}, x^{\prime}\right):=h\left(x_{1}\right)+f\left(x^{\prime}\right) & \text { if }\left(x_{1}, x^{\prime}\right) \in \Omega_{2}:=(-\infty, 0) \times \mathbb{R},\end{cases}
$$

so that $u$ satisfies the conditions $(i)$ and (iii) (which is empty there) of Definition 3.5. Moreover, the function $\nabla u$ is piecewise continuous in $\mathbb{R}^{d}$, and condition (ii) of Definition 3.5 is reduced to

$$
g^{\prime}(0) h^{\prime}(0)>0
$$


Due to the separation of the variables $x_{1}$ and $x^{\prime}$, the gradient flow $X=\left(X_{1}, X^{\prime}\right)$ associated with $\nabla u_{1}$ satisfies

$$
\left\{\begin{aligned}
\frac{\partial X_{1}}{\partial t}\left(t, x_{1}\right) & =g^{\prime}\left(X_{1}\left(t, x_{1}\right)\right) \\
X_{1}\left(0, x_{1}\right) & =x_{1}, \\
\frac{\partial X^{\prime}}{\partial t}\left(t, x^{\prime}\right) & =\nabla_{x^{\prime}} f\left(X^{\prime}(t, x)\right) \\
X^{\prime}\left(0, x^{\prime}\right) & =x^{\prime}
\end{aligned} \quad \text { for } t \in \mathbb{R}, x=\left(x_{1}, x^{\prime}\right) \in \mathbb{R}^{d}\right.
$$

which yields

$$
\left\{\begin{aligned}
X_{1}\left(t, x_{1}\right) & =G^{-1}\left(t+G\left(x_{1}\right)\right) \\
X_{1}\left(0, x_{1}\right) & =x_{1}, \\
\frac{\partial X^{\prime}}{\partial t}\left(t, x^{\prime}\right) & =\nabla_{x^{\prime}} f\left(X^{\prime}(t, x)\right) \\
X^{\prime}(0, x) & =x^{\prime}
\end{aligned} \quad \text { for } t \in \mathbb{R}, x=\left(x_{1}, x^{\prime}\right) \in \mathbb{R}^{d},\right.
$$

where $G^{-1}$ is the inverse function of the primitive $G$ of $1 / g^{\prime}$ in $\mathbb{R}$ such that $G(0)=0$. For a.e. $x \in \mathbb{R}^{d}$, the flow $X(\cdot, x)$ reaches the surface $\left\{x_{1}=0\right\}$ at the time $\tau_{1}(x)=-G\left(x_{1}\right)$ which implies $X_{1}\left(\tau_{1}(x), x_{1}\right)=0$. Then, by Theorem 2.1 and formula (2.24) with $u_{1}$, for any constant $\lambda>0$, the gradient $\nabla u_{1}$ is realizable with the continuous conductivity

$$
\sigma_{1}(x)=\lambda \exp \left(\int_{0}^{-G\left(x_{1}\right)}\left[g^{\prime \prime}\left(X_{1}\left(s, x_{1}\right)\right)+\Delta_{x^{\prime}} f\left(X^{\prime}\left(s, x^{\prime}\right)\right)\right] \mathrm{d} s\right) \quad \text { for } x \in \mathbb{R}^{d}
$$

which using the change of variable $t=X_{1}\left(s, x_{1}\right)=G^{-1}\left(s+G\left(x_{1}\right)\right)$ yields

$$
\sigma_{1}(x)=\lambda \frac{g^{\prime}(0)}{g^{\prime}\left(x_{1}\right)} \exp \left(\int_{0}^{-G\left(x_{1}\right)} \Delta_{x^{\prime}} f\left(X^{\prime}\left(s, x^{\prime}\right)\right) \mathrm{d} s\right) \quad \text { for a.e. } x \in \mathbb{R}^{d} .
$$

Similarly, the gradient $\nabla u_{2}$ is realizable in $\mathbb{R}^{d}$ with the continuous conductivity

$$
\sigma_{2}(x)=\frac{h^{\prime}(0)}{h^{\prime}\left(x_{1}\right)} \exp \left(\int_{0}^{-H\left(x_{1}\right)} \Delta_{x^{\prime}} f\left(X^{\prime}\left(s, x^{\prime}\right)\right) \mathrm{d} s\right) \quad \text { for a.e. } x \in \mathbb{R}^{d}
$$

where $H$ is the primitive of $1 / h^{\prime}$ in $\mathbb{R}$ such that $H(0)=0$. Choosing $\lambda=h^{\prime}(0) / g^{\prime}(0)>0$ by (4.13), we get the flux continuity condition across the interface $\left\{x_{1}=0\right\}$, i.e.

$$
\sigma_{1}\left(0, x^{\prime}\right) \frac{\partial u_{1}}{\partial x_{1}}\left(0, x^{\prime}\right)=\sigma_{2}\left(0, x^{\prime}\right) \frac{\partial u_{2}}{\partial x_{1}}\left(0, x^{\prime}\right)=h^{\prime}(0) \quad \text { for } x^{\prime} \in \mathbb{R}^{d-1}
$$


Therefore, the gradient $\nabla u$ is realizable with the piecewise continuous conductivity

$$
\sigma(x)= \begin{cases}\frac{h^{\prime}(0)}{g^{\prime}\left(x_{1}\right)} \exp \left(\int_{0}^{-G\left(x_{1}\right)} \Delta_{x^{\prime}} f\left(X^{\prime}\left(s, x^{\prime}\right)\right) \mathrm{d} s\right) & \text { if } x \in(0, \infty) \times \mathbb{R}^{d-1} \\ \frac{h^{\prime}(0)}{h^{\prime}\left(x_{1}\right)} \exp \left(\int_{0}^{-H\left(x_{1}\right)} \Delta_{x^{\prime}} f\left(X^{\prime}\left(s, x^{\prime}\right)\right) \mathrm{d} s\right) & \text { if } x \in(-\infty, 0) \times \mathbb{R}^{d-1} .\end{cases}
$$

Acknowledgements. The author is very grateful to the unknown referees for their careful reading and quite relevant comments and references, which have significantly improved the presentation of the paper especially for Section 3.

\section{REFERENCES}

[1] G. Alessandrini, An identification problem for an elliptic equation in two variables. Ann. Mat. Pura Appl. 145 (1986) $265-296$.

[2] G. Alessandrini and V. Nesi, Univalent $\sigma$-harmonic mappings. Arch. Ration. Mech. Anal. 158 (2001) $155-171$.

[3] A. Ancona, Some results and examples about the behavior of harmonic functions and Green's functions with respect to second order elliptic operators. Nagoya Math. J. 165 (2002) 123-158.

[4] F. Bongiorno and V. Valente, A method of characteristics for solving an underground water maps problem. Vol. 116. I.A.C. Publications, Italy (1977).

[5] M. Briane and G.W. Milton, Homogenization of the three-dimensional Hall effect and change of sign of the Hall coefficient. Arch. Ration. Mech. Anal. 193 (2009) 715-736.

[6] M. Briane, G.W. Milton and V. Nesi, Change of sign of the corrector's determinant for homogenization in three-dimensional conductivity. Arch. Ration. Mech. Anal. 173 (2004) 133-150.

[7] M. Briane, G.W. Milton and A. Treibergs, Which electric fields are realizable in conducting materials? ESAIM: M2AN 48 (2014) 307-323.

[8] P.G. Ciarlet, The finite element method for elliptic problems. In Vol. 40 of Classics in Applied Mathematics. Society for Industrial and Applied Mathematics (SIAM), Philadelphia, PA (2002).

[9] R.J. DiPerna and P.-L. Lions, Ordinary differential equations, transport theory and Sobolev spaces. Invent. Math. 98 (1989) $511-547$.

[10] A. Farcas, L. Elliott, D.B. Ingham and D. Lesnic, An inverse dual reciprocity method for hydraulic conductivity identification in steady groundwater flow. Adv. Water Resour. 27 (2004) 223-235.

[11] M.W. Hirsch, S. Smale and R.L. Devaney, Differential Equations, Dynamical Systems, and an Introduction to Chaos, second edition In Vol. 40 of Pure and Applied Mathematics. Elsevier Academic Press, Amsterdam (2004).

[12] C. Kern, M. Kadic and M. Wegener, Experimental evidence for sign reversal of the hall coefficient in three-dimensional metamaterials. Phys. Rev. Lett. 118 (2017) 016601.

[13] I. Knowles, Parameter identification for elliptic problems. J. Comput. Appl. Math. 131 (2001) 175-194.

[14] J.L. Miller, Semiconductor metamaterial fools the Hall effect. Phys. Today 70 (2017) 21-23.

[15] M. Notomi, Materials science: chain mail reverses the Hall effect. Nature 544 (2017).

[16] G.R. Richter, An inverse problem for the steady state diffusion equation. SIAM J. Appl. Math. 41 (1981) $210-221$. 